\title{
HRJ
}

v.3 n.14 (2022)

Recebido: 17/11/2021

Aceito: 06/12/2021

\section{Checklist de cirurgia segura: conhecimento e desafios da equipe de enfermagem}

\author{
Priscila Miranda Carvalho Coletto ${ }^{1}$ \\ GleyceMikaelle Costa Quirino ${ }^{2}$ \\ Lauane Rocha Itacarambi ${ }^{3}$ \\ Ruth Silva Matos ${ }^{4}$ \\ Jacqueline Ramos de Andrade Antunes Gomes ${ }^{5}$ \\ Verônica Santos de Melo ${ }^{6}$ \\ Vitor Francisco Brandão ${ }^{7}$ \\ Osmar Pereira dos Santos 8 \\ TanielaMarquezde Paula ${ }^{9}$ \\ Valine Angelica Borges Batista ${ }^{10}$ \\ Vanessa da Silva Ferreira ${ }^{11}$ \\ Sônia Maria Alves Gomes ${ }^{12}$
}

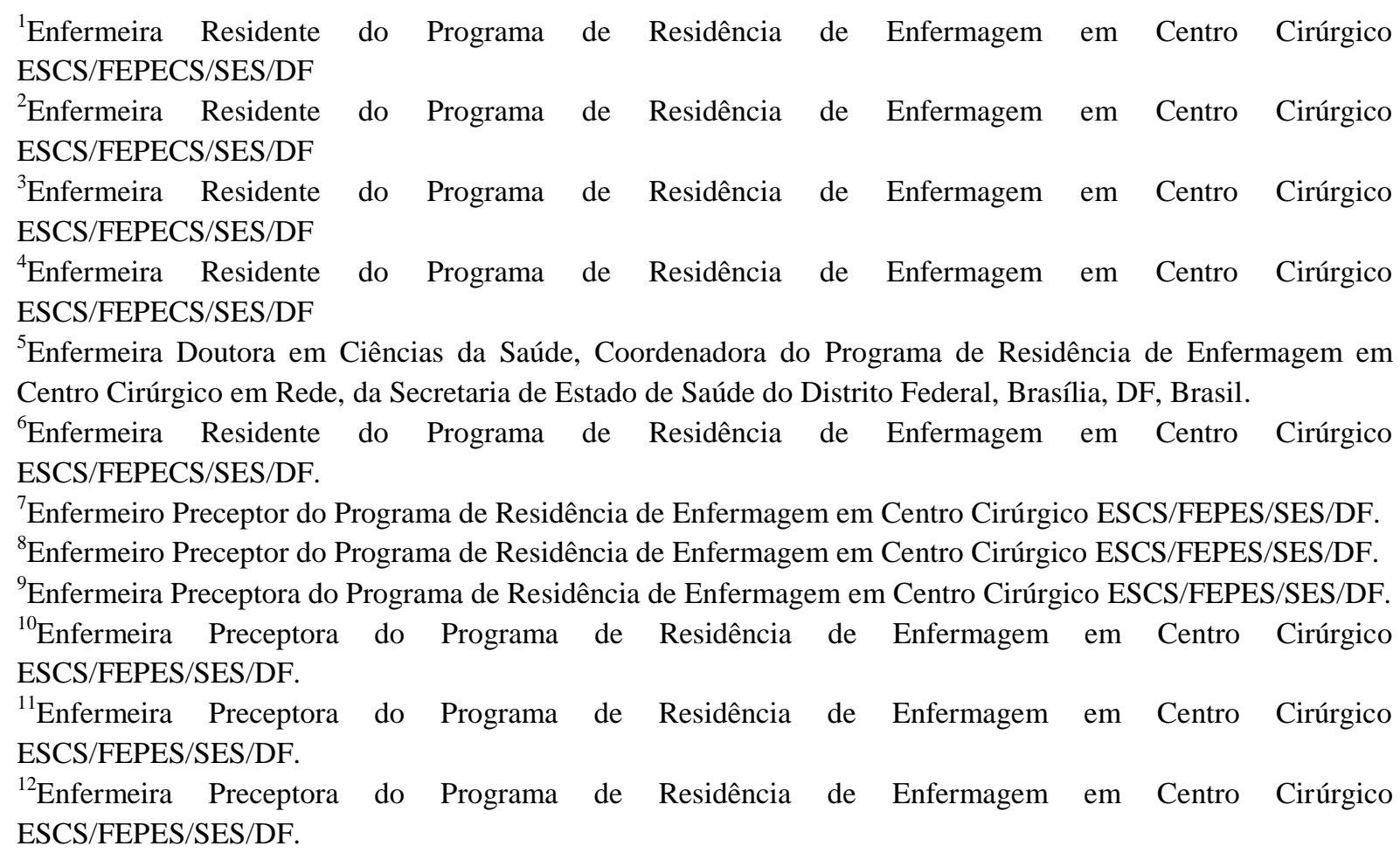

\section{RESUMO}

Objetivo: Analisar a percepção das equipes de enfermagem dos centros cirúrgicos de dois hospitais públicos do Distrito Federal, acerca da importância e benefícios do checklist de cirurgia segura, do treinamento recebido sobre o tema, bem como a segurança e dificuldades 
com o uso do instrumento. Método: Estudo descritivo, exploratório, com abordagem quantitativa. A coleta de dados foi realizada por meio de questionário com escala de Likert, aplicado no mês de janeiro de 2018, totalizando 46 questionários. Resultados: Cerca de $78,3 \%(\mathrm{n}=36)$ da equipe de enfermagem concorda com a importância do checklist e seus benefícios quanto à segurança do paciente e diminuição do índice de infecção do sítio cirúrgico (ISC), apesar de alguns relatos de discordância sobre a diminuição do ISC (17,4\%; n $=8)$. No entanto, parte dos profissionais concordam que estão inabilitados para o uso $(60,9 \%$; $\mathrm{n}=28)$ e sentem insegurança ou dificuldade com checklist $(52,2 \%, \mathrm{n}=24)$. Conclusão: $\mathrm{O}$ presente estudo permitiu identificar falta de compreensão clara sobre os benefícios do checklist e a deficiência de capacitação para seu uso que podem explicar a insegurança e dificuldades identificadas na pesquisa.

Palavras-chaves: Lista de Checagem; Segurança do Paciente; Procedimentos Cirúrgicos Operatórios; Centro Cirúrgico Hospitalar; Pesquisa sobre Serviços de Saúde.

\title{
Safe Surgery Checklist: Nursing Team Knowledge and Challenges
}

\begin{abstract}
Objective: To analyze the perception of nursing staff in surgical centers in two public hospitals in the Federal District, regarding the importance and benefits of the safe surgery checklist, the training received on the subject, as well as the safety and difficulties with the use of the instrument. Method: Descriptive, exploratory study with a quantitative approach. Data collection was performed using a Likert scale questionnaire, applied in January 2018, totaling 46 questionnaires. Results: About $78.3 \%(n=36)$ of the nursing staff agree on the importance of the checklist and its benefits in terms of patient safety and decrease in the surgical site infection rate (SSI), despite some reports of disagreement on the decrease in SSI $(17.4 \% ; \mathrm{n}=8)$. However, some professionals agree that they are unable to use it $(60.9 \% ; \mathrm{n}=$ $28)$ and feel insecure or have difficulty with the checklist $(52.2 \%, \mathrm{n}=24)$. Conclusion: This study allowed us to identify a lack of clear understanding about the benefits of the checklist and the lack of training for its use, which may explain the insecurity and difficulties identified in the research.
\end{abstract}

Keywords: Checklist; Patient safety; Operative Surgical Procedures; Hospital Surgical Center; Health Services Survey

\section{INTRODUÇÃO}

Dados sugerem que milhões de pacientes submetidos a procedimentos cirúrgicos sofrerão uma complicação pós-operatória, levando a inúmeros óbitos por ano ${ }^{1}$. Estima-se que 1 em 150 pacientes admitidos em estabelecimento de saúde vai a óbito em consequência de eventos adversos, e que cerca de dois terços desses eventos estão associados a procedimentos

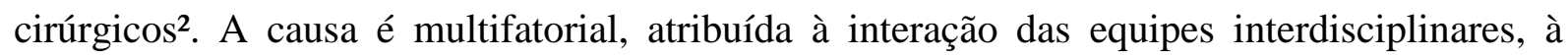
complexidade dos procedimentos e ao trabalho sob pressão ${ }^{1}$. No entanto, a maioria dos riscos que o centro cirúrgico pode oferecer, poderia ser evitada ${ }^{3}$. 
A infecção de sítio cirúrgico (ISC) é a complicação mais frequente do paciente operado ${ }^{4}$. No Brasil, a ISC ocupa a terceira posição entre todas as infecções em serviços de saúde, compreendendo de $14 \%$ a $16 \%{ }^{(5-6)}$.

Em 2009, a Organização Mundial da Saúde (OMS) lançou o programa "Cirurgias seguras salvam vidas", com objetivo aumentar os padrões de qualidade da assistência cirúrgica em serviços de saúde de todo o mundo ${ }^{7}$. É considerado um marcador de substituição para a qualidade do atendimento ${ }^{8}$. A OMS incentiva a adoção da lista de verificação de segurança cirúrgica, o checklist, com checagem verbal dos seus itens pela equipe multidisciplinar na sala cirúrgica ${ }^{9}$. Assim, a estratégia para efetivar melhorias na assistência cirúrgica foi a adesão desse checklist de cirurgia segura, composto de três etapas, sendo elas: identificação (antes da indução anestésica), confirmação (antes da incisão cirúrgica) e registro (antes de o paciente sair da sala cirúrgica $)^{10}$. O resultado de uma avaliação em oito instituições no mundo mostra que o uso do checklist reduziu $47 \%$ da mortalidade e complicações ${ }^{11}$.

A implementação do checklist é de baixo custo, resumindo-se na reprodução e distribuição do instrumento. Estima-se que é necessário um tempo total de três minutos para a aplicação das três fases. Porém, a dificuldade de aplicação está localizada na equipe cirúrgica $^{12}$. A finalidade e o preenchimento correto do checklist devem ser demonstrados à equipe durante o período de implantação ${ }^{13}$.

No Brasil, experiências relacionadas ao checklist apresentam muitos erros, tais como baixa adesão, checagem sem verbalização, não preenchimento de itens, ausência da equipe completa na checagem, e resistência do uso pelos profissionais ${ }^{9}$. Não basta que as instituições implantem os protocolos, é necessário que os profissionais façam o uso correto da ferramenta. Isso ocorre quando as equipes compreendem a importância e a necessidade da prática para o sucesso de sua aplicação ${ }^{14}$. 
Acredita-se que a falta de conhecimento e capacitação das equipes de enfermagem a respeito do tema, bem como aspectos aliados à grande demanda de funções e às precariedades vistas no serviço público de saúde, possam estar exercendo influência negativa na adesão e no uso correto da lista de verificação de segurança cirúrgica ${ }^{15}$.

Logo, a realização de estudos sobre a percepção da equipe de enfermagem em relação ao instrumento pode servir de apoio na formulação de explicações que impedem o uso correto. Consequentemente, também pode servir de apoio na formulação de estratégias de melhoria. Nesse sentido, o presente estudo teve como objetivo analisar a percepção das equipes de enfermagem de dois centros cirúrgicos de hospitais públicos do Distrito Federal (DF), acerca da importância e benefícios do checklist de cirurgia segura, do treinamento recebido sobre o tema, bem como a segurança e dificuldades com o uso do instrumento.

\section{METODOLOGIA}

Trata-se de um estudo descritivo, exploratório, com abordagem quantitativa. A pesquisa foi desenvolvida nos centros cirúrgicos de dois hospitais públicos do Distrito Federal no mês de janeiro de 2018. Os hospitais estão localizados na região sul do DF, a saber: Hospital Regional de Santa Maria e Hospital Regional do Gama, referências de média e alta complexidade para o distrito federal que atendem cirurgias de pequeno, médio e grande porte, abrangendo 26 especialidades.

A população do estudo foi constituída pela equipe de enfermagem composta por enfermeiros, técnicos e auxiliares de enfermagem. Os participantes foram convidados, em seu local de trabalho, a participar da pesquisa. Informava-se a finalidade da pesquisa e solicitavase sua colaboração. Fizeram parte da amostra 46 profissionais que atuam no centro cirúrgico nos dois períodos de trabalho, dia e noite, tendo somente como critérios de inclusão pertencer à equipe de enfermagem daquela unidade e livre concordância para participar do estudo. 
Para coleta de dados foi desenvolvido um questionário estruturado (Anexo) constituído por duas partes: a primeira contendo os dados sócio demográficos dos participantes (idade, escolaridade, tempo de atuação na função, acumulação de empregos e carga horária semanal total) e a segunda com sete proposições referentes a percepção dos entrevistados referente a importância e benefícios do checklist, o treinamento recebido e o nível de segurança e dificuldade como uso do instrumento.

O questionário foi construído com base escala de Likert de 5 pontos ${ }^{16}$ na qual os entrevistados especificam seu nível de concordância com uma afirmação. Para cada pergunta o entrevistado tinha as seguintes opções: discordo totalmente (DT), discordo parcialmente (DP), não concordo e nem discordo (I), concordo parcialmente (CP) e concordo totalmente (CT). Foram obtidas as frequências e o ranking médio (RM) para cada proposição. Foram somados os valores das respostas dos entrevistados para cada assunto abordado (importância do checklist, treinamento recebido e segurança e dificuldade com o uso do instrumento) e as pontuações foram classificadas em três níveis de concordância (alto, moderado e baixo). Os dados foram analisados utilizando o software IBM SPSS Statistics versão 20.

A pesquisa foi aprovada pelo Comitê de Ética em Pesquisa da Fundação de Ensino e Pesquisa em Ciências da Saúde (FEPECS), sob número do CAAE 81703917.6.0000.5553.

\section{RESULTADOS}

Por meio de uma abordagem quantitativa, este estudo avaliou a percepção das equipes de enfermagem acerca do checklist de cirurgia segura em dois hospitais públicos. Os resultados foram baseados na análise estatística das respostas das equipes de enfermagem. Os aspectos abordados com os entrevistados incluíram suas percepções acerca da importância do 
checklist, do treinamento recebido bem como do nível de segurança e dificuldade com o instrumento.

\section{Características da equipe de enfermagem}

Tratou-se de uma população adulta, ou seja, apresentou uma idade média igual a 43,9 $\pm 6,73$ anos, que variou de 27 a 58 anos. A análise do tempo de exercício profissional desse grupo demonstrou uma média de 16,1 \pm 6,95 anos e de atuação em centro cirúrgico a média foi de $11,1 \pm 6,82$ anos. No que se refere ao acúmulo de empregos, $23,9 \%$ dos profissionais afirmaram ter outro vínculo. A figura 1 apresenta a média e desvio padrão da idade dos entrevistados, o tempo de atuação na função e o tempo de atuação em centro cirúrgico em anos.

Figura 1: Média e desvio padrão da idade, tempo de atuação na função e no centro cirúrgico expressos em anos, e carga horária semanal de trabalho expressa em horas. $(n=46)$.

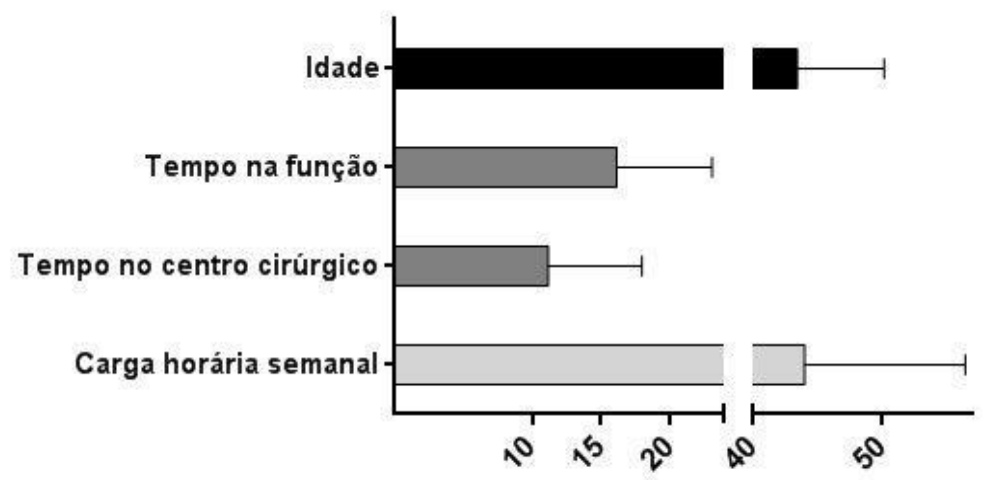

Fonte: Elaborado pela própria autora.

Em termos de função exercida, a maioria dos profissionais entrevistados era técnica de enfermagem $(80,4 \%, n=37)$. Cerca de $15,2 \%(n=7)$ eram enfermeiros e 4,3\% $(n=2)$ auxiliares de enfermagem; desses, 65\% $(n=30)$ tem ensino superior e $32,5 \%(n=15)$ tem o ensino médio. Os dados se encontram na figura 2. 
Figura 2: Frequência (n) de cada função exercida no hospital (enfermeiro, técnico de enfermagem e auxiliar de enfermagem), do grau de escolaridade (ensino médio, ensino superior e pós-graduação) e funcionários que fazem acumulação de empregos ou não.

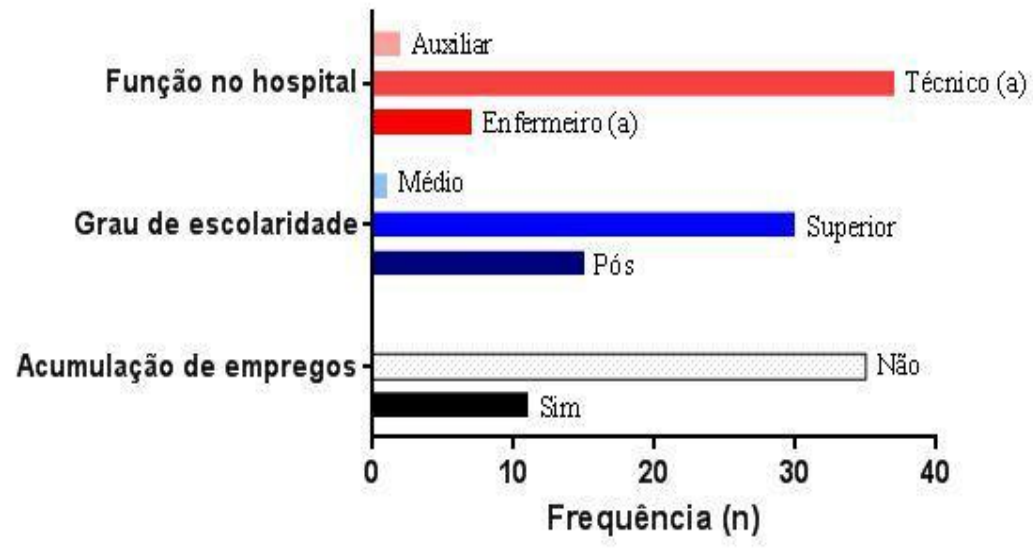

Fonte: Elaborado pela própria autora.

\section{Percepção da equipe perante o checklist}

Um dos aspectos essenciais da percepção dos profissionais a respeito do checklist de cirurgia segura é referente à importância e benefícios do instrumento. De maneira geral, valores expressivos foram observados pelos entrevistados quanto ao assunto (Tabela 1). Dos profissionais entrevistados, $89,1 \%(n=41)$ concordaram totalmente que conhecem a importância do checklist e poucos discordam da proposição $(4,4 \%, \mathrm{n}=2)$, conforme descrito na tabela 1. Isso mostra que os profissionais estão conscientes da necessidade de aplicação do protocolo e que esse faz parte do cotidiano deles.

Tabela 1. Resultados relacionados à percepção sobre a importância do checklist.

Proposições sobre a importância do Escala de Likert* checklist 
$\mathrm{O}$ checklist traz maior segurança para o

atendimento paciente e evita os eventos adversos.

$4,3 \quad 4,3 \quad 4,3 \quad 10,9 \quad 80,4 \quad 4,5$

Concordo que o uso correto do checklist pode diminuir o índice de infecção do sítio cirúrgico (ISC).

$10,9 \quad 6,5 \quad 13,0 \quad 6,5 \quad 63,0 \quad 4,0$

* Expressão em percentagem (\%); RM - Ranking Médio.

Fonte: Elaborado pela própria autora.

Quanto aos benefícios que o instrumento pode trazer, $80,4 \%(\mathrm{n}=37)$ concordaram totalmente que o checklist traz maior segurança e evita eventos adversos. Por outro lado, quando questionados a respeito de concordarem que o uso correto do checklist pode diminuir o índice de infecção do sítio cirúrgico (ISC), observaram-se resultados menores de concordância $(63 \%, \mathrm{n}=29)$. Diante disso, foi visto que parte dos entrevistados não conhecem este benefício do checklist, pois 17,4\% $(\mathrm{n}=8)$ discordaram e 13\% $(\mathrm{n}=6)$ não concordaram e nem discordaram.

Apesar do menor conhecimento da equipe quanto aos benefícios do checklist no ISC, foi calculado um nível de percepção da equipe em relação a importância e os benefícios do checklist no geral. Foi verificado que 78,3\% $(\mathrm{n}=36)$ dos profissionais obtiveram alta pontuação (12 a 15 pontos na soma das três proposições da tabela 1). Isso significa que grande parte dos profissionais considerou o checklist muito importante e concordaram com seus benefícios.

Também foram aplicadas proposições referentes ao treinamento que os profissionais tiveram sobre o preenchimento do instrumento (Tabela 2). É provável que o treinamento influencie no uso correto do checklist pelas equipes. A proposição afirmava que receberam treinamento específico para o preenchimento do checklist e uma frequência considerável de entrevistados discordaram $(37 \%, \mathrm{n}=17)$. Vale ressaltar também que, $15,2 \%(\mathrm{n}=7)$ não 
concordaram e nem discordaram com a afirmação. Ao classificar como ótima a orientação que tiveram no treinamento, foi observado um valor expressivo de discordância com a afirmativa, cerca de 47,8\% $(n=22)$ discordaram parcialmente ou totalmente.

Tabela 2.Resultados relacionados à percepção sobre o treinamento recebido para ochecklist.

\begin{tabular}{|c|c|c|c|c|c|c|}
\hline \multirow{2}{*}{ Proposições sobre o treinamento recebido } & \multicolumn{5}{|c|}{ Escala de Likert $*$} & \multirow{2}{*}{$\mathrm{RM}$} \\
\hline & DT & DP & I & $\mathrm{CP}$ & $\mathrm{CT}$ & \\
\hline \multicolumn{7}{|l|}{$\begin{array}{l}\text { Recebi treinamento específico para preencher } \\
\text { o checklist de segurança cirúrgica }\end{array}$} \\
\hline & 28,3 & 8,7 & 15,2 & 13,0 & 34,8 & 3,2 \\
\hline \multicolumn{7}{|l|}{$\begin{array}{l}\text { Classifico como ótima a orientação que recebi sobre } \\
\text { como preencher o checklist. }\end{array}$} \\
\hline & 34,8 & 13,0 & 10,9 & 23,9 & 17,4 & 2,76 \\
\hline
\end{tabular}

Quando calculado o nível de concordância dos entrevistados em relação ao treinamento que receberam, 60,9\% $(\mathrm{n}=28)$ obtiveram pontuação baixa ou moderada (até 7 pontos na soma das duas proposições da tabela 2). Consideramos que esses profissionais concordaram que estão pouco ou mal treinados.

Ao analisar como os entrevistados se sentem ao utilizar o checklist (Tabela 3), verificou-se que apenas $30,4 \%(n=14)$ concordaram totalmente que se sentem seguros em preencher todos os itens instrumento. Apenas $26,1 \%(n=12)$ discordaram totalmente com a afirmativa. 
Tabela 3. Resultados relacionados à percepção sobre o sentimento de segurança e dificuldade no uso do checklist.

Proposições sobre segurança e dificuldade em usar o checklist

Escala de Likert*

$\mathrm{RM}$

DT DP I CP CT

$26,1 \quad 10,9 \quad 10,9 \quad 21,7 \quad 30,4 \quad 3,2$

Sinto-me totalmente seguro em fazer o checklist

Não tenho dificuldade em preencher nenhum item do checklist.

$21,7 \quad 8,7 \quad 13,0 \quad 17,4 \quad 39,1 \quad 3,4$

* Expressão em percentagem (\%); RM - Ranking Médio.

Sobre não sentir dificuldade no preenchimento de nenhum item do checklist, um valor expressivo de profissionais discordou $(30,4 \%, \mathrm{n}=14)$ e cerca de $13,0 \%(\mathrm{n}=6)$ não concordaram e nem discordaram da afirmativa. Esses resultados demonstram que boa parte da equipe de enfermagem apresenta dificuldades no preenchimento.

Quanto ao nível de segurança e dificuldade dos profissionais em relação ao checklist, obtido pela soma das pontuações na escala de Likert com as duas proposições sobre o assunto, mais da metade dos entrevistados $(52,2 \%, \mathrm{n}=24)$ foram classificados com baixa ou moderada pontuação de concordância (até 7 pontos na soma das duas proposições da tabela 3). Consideramos que essas pessoas sentem algum tipo de insegurança ou dificuldade com o uso do checklist.

\section{DISCUSSÃO}

O presente estudo limitou-se pela dificuldade de comunicação com os profissionais que fazem uso do checklist. Por isso, tratou-se de uma amostra pequena, que pode não ter trazido resultados confiáveis que representem a população. A ausência de local apropriado 
para entrevista e os frequentes relatos de falta de tempo hábil para responder o questionário podem ter levado os entrevistados a não responderem direito ao questionário, levando a respostas ambíguas ou não verdadeiras.

Em 2009, por meio do New England Journal of Medicine, foram publicados os primeiros resultados da implementação do checklist do programa 'Cirurgias Seguras Salvam Vidas', sendo avaliados, inicialmente, apenas dois parâmetros: grandes complicações e mortalidade. Ambos os parâmetros apresentaram quedas significativas, comprovando a efetividade da utilização do checklist para o aumento da segurança assistencial ${ }^{17}$. No entanto, a forma como esse checklist é aplicado, conhecimento e capacitação da equipe são necessários para que ele alcance sua eficácia ${ }^{15}$. Assim, acredita-se que este estudo possa direcionar as atividades desenvolvidas dos hospitais avaliados, visando buscar melhorias para o uso correto do instrumento e contribuir para novas pesquisas e publicações referentes à temática.

Apesar de grande parte dos profissionais que responderam o questionário exercer uma função de nível médio (80,4\% de técnicos de enfermagem), a maioria apresentou nível superior de escolaridade (65\%) e mais de 10 anos de atuação no centro cirúrgico. Por isso, esperava-se que todos os profissionais conhecessem e entendessem a importância do checklist , visto que o protocolo já foi implantado nas instituições há quase dez anos e que são conhecidas em todo mundo as evidências sobre seus benefícios ${ }^{3}$. No entanto, dos profissionais entrevistados, ainda foi encontrado alguns (21\%) que consideraram o checklist pouco importante ou de importância moderada (pontuação na escala Likert 7 a 11).

Para tentar explicar essa frequência de entrevistados que pareceram não entender completamente a importância do checklist, podem-se analisar os resultados das três proposições acerca do assunto. Foi observada uma ocorrência de 14 entrevistados que não concordaram com a terceira proposição. Nessa, há uma afirmativa de que o uso correto do checklist pode diminuir o índice de Infecção do Sítio Cirúrgico (ISC). O ISC é a complicação 
mais frequente do paciente operado e contribui com cerca de $31 \%$ das infecções relacionadas a assistência à saúde. ${ }^{4} \mathrm{O}$ checklist é um instrumento importante na prevenção e tratamento do ISC, causando diminuição desses índices. ${ }^{6}$ Portanto, a falta de concordância sobre esse benefício pode explicar a ocorrência de alguns entrevistados que consideraram o instrumento pouco importante ou de importância moderada, fato que pode contribuir para uma negligência do uso do instrumento por parte da equipe.

É de extrema importância e necessidade que os profissionais conheçam a importância do checklist para uma aplicação qualificada do instrumento. Os pacientes expostos a uma lista de verificação de segurança cirúrgica experimentam melhores resultados pós-operatórios, mas isso só ocorre diante do bom uso do instrumento e em hospitais onde o checklist faz parte da rotina. ${ }^{3}$

Pode-se afirmar que as equipe analisadas consideraram que não receberam um treinamento bom e específico sobre o checklist. As proposições a respeito do nível de treinamento mostraram que mais da metade dos participantes opinaram negativamente, sendo que, $37 \%$ consideram-se mal treinados e $29,9 \%$ sem opinião ou consideram-se pouco treinados.

Ao avaliar os resultados de como os participantes perceberam seus sentimentos em relação ao checklist, determinando um nível de segurança e dificuldade com o instrumento, verificou-se um número expressivo de entrevistados que se sentem inseguros e com dificuldades, ou com pouca segurança e dificuldade, somando um total de 52,2\%. Nossos resultados sobre a percepção da equipe quanto à importância, benefícios e treinamento recebido sobre o checklist, podem explicar essa insegurança e dificuldade. A falta ou pouca compreensão do uso, importância e benefícios do checklist pode ser uma dificuldade enfrentada pelas equipes de enfermagem, bem como a falta de capacitação. 
Em um estudo realizado por enfermeiras em unidades hospitalares do Distrito Federal, consideram que as maiores barreiras para o correto funcionamento do checklist são a falta de treinamento da equipe, a não adesão dos profissionais ao protocolo e o não comprometimento da instituição ${ }^{18}$. Sobre tais situações, é provável que essas também sejam dificuldades enfrentadas nos dois hospitais incluídos no presente estudo.

Uma intervenção no sentido de sanar as dúvidas das equipes perante o checklist, com uma capacitação continuada, seria uma alternativa para o funcionamento efetivo do instrumento. Sendo o centro cirúrgico considerado como cenário de alto risco, onde os processos de trabalho constituem-se em práticas complexas, interdisciplinares, com forte dependência da atuação individual e da equipe em condições ambientais dominadas por pressão e estresse, é indispensável a orientação e treinamento dos envolvidos na prática do centro cirúrgico quando houver implementação de um novo protocolo ${ }^{19}$.

Ainda sobre a dificuldade da equipe de enfermagem com o checklist identificada em nosso estudo, pode ter havido influência do conhecimento de outros profissionais do centro cirúrgico em relação ao checklist, o que influencia no uso correto do instrumento. Uma única pessoa deve ser responsável pela verificação que frequentemente será um profissional de enfermagem. Contudo, o preenchimento do checklist depende da colaboração e informação fornecida por outros profissionais ${ }^{19}$. O estudo abrangeu a equipe de enfermagem, mas foi observado durante a aplicação do questionário, que esses profissionais se queixaram de muitos médicos que não conhecem o checklist e relataram que isso dificulta o preenchimento.

Durante a aplicação do questionário, também se observou que a equipe de enfermagem apresenta dificuldade em entender a importância da realização da checagem, pois alguns profissionais relataram não ter tempo suficiente para preencher nos momentos corretos, mas que entregam o instrumento preenchido, sendo que é necessário que seja preenchido nos momentos preconizados pelo protocolo da $\mathrm{OMS}^{20}$. 
Embora os resultados não permitam generalizações tão amplas, pondera-se que os mesmos fornecem um panorama das limitações cotidianas enfrentadas tais como, não ter recebido treinamento específico para o cheklist, bem como ter de dificuldade com o instrumento. É imprescindível que sejam realizadas capacitações com todos os profissionais que irão atuar com o instrumento, antes de ser implementado e mesmo depois, com educação continuada. É necessário mostrar à equipe o porquê de estarem trabalhando com este protocolo e como fazer o uso correto, do contrário eles não estarão aptos para usá-lo ${ }^{20}$. Ademais, é importante salientar que, receber capacitação específica do checklist por si só, não garante resultados positivos, pois requer o comprometimento integral e incondicional de todos os profissionais que atuam no setor.

\section{CONCLUSÃO}

Foi possível alcançar o objetivo de analisar a percepção das equipes de enfermagem acerca da importância e benefícios do checklist de cirurgia segura, do treinamento recebido sobre o tema, bem como a segurança e dificuldades com o uso do instrumento. Parte da equipe de enfermagem concorda com a importância do checklist e os seus benefícios quanto à segurança do paciente e diminuição do índice de infecção do sítio cirúrgico (ISC), apesar de alguns relatados de discordância sobre a diminuição do ISC. No entanto, a maior parte dos profissionais concordou que estão pouco ou mal treinados e sentem algum tipo de insegurança ou dificuldade com o uso do checklist. O presente estudo permitiu identificar que uma ligeira falta de compreensão clara sobre os benefícios do checklist e a falta de capacitação para seu uso podem explicar a insegurança e dificuldades identificadas no estudo. 


\section{REFERÊNCIAS}

1. Weiser TG, Haynes AB, Molina G, Lipsitz SR, Esquivel MM, Uribe-Leitz T, Fu R, Azad T, Chao T, Berry WR, Gawande AA. Estimate of the global volume of surgery in 2012: an assessment supporting improved health outcomes. Lancet. 2015;385 Suppl 2: S11. Doi: 10.1016/S0140-6736(15)60806-6.

Disponível

em:

\section{https://pubmed.ncbi.nlm.nih.gov/26313057/}

2. Secanell M, Orrego C, Vila M, Vallverdú H, Mora N, Oller A, Bañeres J. A surgical safety checklist implementation: experience of a start-up phase of a collaborative project in hospitals of Catalonia, Spain. Med Clin (Barc). 2014; 143 Suppl 1:17-24. doi:10.1016/j.medcli.2014.07.007.

em:

\section{https://pubmed.ncbi.nlm.nih.gov/25128355/}

3. Abbott TEF, Ahmad T, Phull MK, Fowler AJ, Hewson R, Biccard BM, Chew MS, Gillies M, Pearse RM. The surgical safety checklist and patient outcomes after surgery: a prospective observational cohort study, systematic review and meta-analysis. Br J Anaesth. 2018; 120(1):146-155. doi:10.1016/j.bja.2017.08.002.

Disponível

em:

\section{https://www.bjanaesthesia.org/article/S0007-0912(17)53945-8/fulltext}

4. Bellusse GC, Ribeiro JC, Campos FR, Poveda VB, Galvão CM. Fatores de risco de infecção da ferida operatória em neurocirurgia. Acta Paul Enferm [periódico na Internet]. 2015. [acessado 2021 Mai 31]28(1):66-73. https://doi.org/10.1590/1982-0194201500012. Disponível em:

\section{https://www.scielo.br/j/ape/a/PvKnfdb65XmLgjD8s4QvhXx/?format=pdf\&lang=pt}

5. Agência Nacional de Vigilância Sanitária (ANVISA). Critérios Diagnósticos de Infecção Relacionada à Assistência à Saúde - Série Segurança do Paciente e Qualidade em Serviços de Saúde. $\quad$ 80p. 2013; Disponível em: $\underline{\text { www20.anvisa. }}$ gov.br/segurancadopaciente/index.php/publicacoes/category/livros. 
6. Roscani ANCP, Ferraz EM, Filho AGO, Freitas MIP. Validação de checklist cirúrgico para prevenção de infecção de sítio cirúrgico. Acta Paul Enferm. [periódico na Internet]. 28 (6) • Nov-Dec 2015 [acessado 2021 Mai 31]; https://doi.org/10.1590/1982-0194201500092 Disponível em:

\section{https://www.scielo.br/j/ape/a/gM6ggmbdbCfKCCLqRJLqmrQ/?format=pdf\&lang=pt}

7. Weiser TG, Regenbogen SE, Thompson KD, Haynes AB, Lipsitz SR, Berry WR, Gawande AA. An estimation of the global volume of surgery: a modelling strategy based on available data. Lancet. [periódico na Internet]. 2008 [acessado 2021 Mai 31];372(9633):139-144. Doi: 10.1016/S0140-6736(08)60878-8. Disponível em: https://www.thelancet.com/journals/lancet/article/PIIS0140-6736(15)60806-6/fulltext

8. Bergs J, Hellings J, Cleemput I, Zurel O, Troyer V, Hiel MV, Demeere JL, Claeys D, Vandijck D. Revisão sistemática e meta-análise do efeito da lista de verificação de segurança cirúrgica da Organização Mundial da Saúde sobre complicações pós-operatórias. Br J Surg. [periódico na Internet].2014 [acessado 2021 Mai 31]; 101: 150-158. https://doi.org/10.1002/bjs.9381. Disponivel em: https://pubmed.ncbi.nlm.nih.gov/26313057/

9. Tostes MFP, Haracemiw A, MAI LD. Lista de verificação de segurança cirúrgica: Considerações a partir da micropolítica institucional. Esc Anna Nery [periódico na Internet]. 2016 [acessado 2021 Mai 31];20(1):203-209. DOI: 10.5935/1414-8145.20160027. Disponível em:

https://www.scielo.br/j/ean/a/p7NxCCLCs57vPv38RzMHffj/abstract/?format=html\&stop=ne $\underline{\mathrm{xt} \& \text { lang }=\mathrm{pt}}$

10. Sucupira E, Matta R, Zuker P, Matta J, Arbeláez JP, Uebel CO. Lista de verificação para cirurgia plástica estética: uma ferramenta de segurança. Cirurgia Plástica Estética. [periódico na Internet]. Outubro de 2016 [acessado 2021 Mai 31]; 40 (5): 785-791. DOI: 10.1007 / 
s00266-016-0685-y. Disponível em: https://link.springer.com/article/10.1007/s00266-016$\underline{0685-y}$

11. Pancieri AP, Santos BP, Avila MAG, Braga EM. Checklist de cirurgia segura: análise da segurança e comunicação das equipes de um hospital escola. Rev. Gaúcha Enferm. [periódico na Internet]. 34 (1) • Mar 2013 [acessado 2021 Mai 31]; https://doi.org/10.1590/S198314472013000100009. Disponível em: https://www.scielo.br/j/rgenf/a/hpcybZ8fkZ8MfxmhWgMccQC/?lang=pt

12. Freitas MR, Antunes AG, Lopes BNA, Fernandes FC, Monte LC, Gama ZAS. Avaliação da adesão ao checklist de cirurgia segura da OMS em cirurgias urológicas e ginecológicas, em dois hospitais de ensino de Natal, Rio Grande do Norte, Brasil. Cad. Saúde Pública 30 (1) [periódico na Internet]. Jan 2014 [acessado 2021 Mai 31]; https://doi.org/10.1590/0102$311 X 00184612$.

Disponível

em: https://www.scielo.br/j/csp/a/XRq3xPH7jMMMgXPSkkkqHSN/?lang=pt

13. Maziero ECS, Silva AEBC, Mantovani MF, Cruz EDA. Adesão ao uso de um checklist cirúrgico para segurança do paciente. Rev Gaúcha Enferm. [periódico na Internet].2015 [acessado 2021 Mai 31];36(4):14-20. https://doi.org/10.1590/1983-1447.2015.04.53716. Disponível em: https://www.scielo.br/j/rgenf/a/tH7s VkqgZLvMGM4rGB8CbCf/?lang=pt

14. Oliveira VM, Piekala DM, Deponti GN, Batista DCR, Minossi SD, Chisté M, Bairros PMN, Naue WS, Welter DI, Vieira SRR. Checklist da prona segura: construção e implementação de uma ferramenta para realização da manobra de prona. Rev. bras. ter. intensiva 29 (2) [periódico na Internet]. Apr-Jun 2017 [acessado 2021 Mai 31]; https://doi.org/10.5935/0103507X.20170023.

Disponível em: https://www.scielo.br/j/rbti/a/MMqL3GT45ydGVYJXKtgVLkb/?lang=pt\&format=pdf

15. Oliveira RM, Leitão IMTA, Silva LMS, Figueiredo SV, Sampaio RL, Gondim MM. Estratégias para promover segurança do paciente: da identificação dos riscos às práticas 
baseadas em evidências. Esc Anna Nery 18 (1) [periódico na Internet]. Jan-Mar 2014 [acessado 2021 Mai 31]; https://doi.org/10.5935/1414-8145.20140018. Disponível em: https://www.scielo.br/j/ean/a/cgFQTChp95c35PvWrp3D4JL/?lang=pt\&format=pdf

16. Likert, R., \& Likert, J. G. New ways of managing confl ict. New York: McGrawHill; 1976.

17. Martins GS, Carvalho R. Realização do timeout pela equipe cirúrgica: facilidades e dificuldades. Rev. SOBECC [periódico na Internet]. São Paulo. 2014 [acessado 2021 Mai 31]; 19(1): 18-25. http://dx.doi.org/10.4322/sobecc.2014.007. Disponível em: https://www.researchgate.net/publication/274692369_Realizacao_do_timeout_pela_equipe_ci rurgica_facilidades_e_dificuldades

18. Carvalho PA, Göttems LBD, Pires MRGM, Oliveira MLC. Safety culture in the operating room of a public hospital in the perception of healthcare professionals. Rev. Latino-Am. Enfermagem 23 (6) [periódico na Internet]. Nov-Dec 2015. https://doi.org/10.1590/01041169.0669.2647. Disponível em: https://www.scielo.br/j/rlae/a/MJkrBpBLFXdkL3g5bctXqGf/abstract/?lang=pt

19. Tiferes J, Bisantz AM, Guru KA. Team interaction during surgery: a systematic review of communication coding schemes. J Surg Res. [periódico na Internet]. 2015 [acessado 2021 Mai 31]; 195(2):422-32. Doi: 10.1016/j.jss.2015.02.034. Disponível em: https://pubmed.ncbi.nlm.nih.gov/25819771/

20. Alpendre FT, Cruz RDA, Dyniewicz AM, Mantovani MF, Silva AEBC, Santos GS. Cirurgia segura: validação de checklist pré e pós-operatório. Rev. Latino-Am. Enfermagem 25, 2017 [acessado 2021 Mai 31]; https://doi.org/10.1590/1518-8345.1854.2907. Disponível em: $\underline{\text { https://www.scielo.br/j/rlae/a/jSgwyyQJpGvyYvV8VmWVKws/?lang=pt\&format=pdf }}$ 